\title{
Evaluation of a high spatial resolution temperature compensated distributed strain sensor using a temperature controlled strain rig
}

\author{
Mohammad Belal ${ }^{1}$ and Trevor P Newson ${ }^{1}$ \\ ${ }^{1}$ ORC, University of Southampton, Southampton, SO17 1BJ, UK
}

\begin{abstract}
We demonstrate a scheme which allows for temperature corrected distributed strain measurements under environments involving simultaneous application of strain and temperature, with enhanced spatial $(5 \mathrm{cms})$, strain $(66 \mu \varepsilon)$ and temperature resolutions $\left(1.9^{\circ} \mathrm{C}\right)$. The technique utilizes the combination of frequency based BOCDA with Brillouin intensity measurements.
\end{abstract}

Keyword: Brillouin scattering, distributed fiber sensors

\section{INTRODUCTION}

In situations where both strain and temperature are simultaneously influencing the same section of the sensing fiber, Brillouin frequency measurements alone are insufficient to unambiguously determine the strain. A solution to this problem has been previously provided ${ }^{[1]}$, but to date has only been tested for sensing rigs where sections have either been heated or strained. This is due to the difficulty of heating an extended strain region. With the advent of techniques allowing high spatial resolution, albeit over shorter sensing lengths, the concept of discriminating temperature and strain can be tested for regions of sensing fiber which are simultaneously strained and heated in an environmental chamber. Brillouin optical correlation domain analysis (BOCDA), whilst offering unrivalled high spatial resolution of the Brillouin frequency shift ${ }^{[2]}$, requires an additional measurement to allow temperature and strain effects to be separated. This paper provides a solution to this problem by combining BOCDA with a distributed measurement of the power of the antiStokes Brillouin scattering (B-OTDR) with very much higher spatial resolution than previously reported, table 1, in order to produce an improved high spatial resolution, fully temperature compensated distributed strain sensor. High spatial resolution Brillouin intensity measurements require very short pulses with high peak powers. Such a requirement ties in well for short range distributed sensing as much higher peak pulses are permissible before non linear deleterious effects such as self phase modulation, cross phase modulation, stimulated Raman scattering become significant.

\begin{tabular}{|l|l|l|l|l|}
\hline Ref. & $\begin{array}{l}\text { Spatial } \\
\text { Resolution }\end{array}$ & Strain Resolution & $\begin{array}{l}\text { Temperature } \\
\text { Resolution }\end{array}$ & Range \\
\hline$[1]$ & $20 \mathrm{~m}$ & $100 \mu \varepsilon$ & $4^{\circ} \mathrm{C}$ & $30 \mathrm{Km}$ \\
\hline$[3]$ & $5 \mathrm{~m}$ & $85 \mu \varepsilon$ & $3.5^{\circ} \mathrm{C}$ & $50 \mathrm{Km}$ \\
\hline$[4]$ & $1.3 \mathrm{~m}$ & $80 \mu \varepsilon$ & $3^{\circ} \mathrm{C}$ & $6.3 \mathrm{Km}$ \\
\hline
\end{tabular}

Table 1. The achievements of previous techniques with spatial, temperature and strain resolutions respectively

In BOCDA a frequency swept laser output is split into two. One of which is frequency shifted by an amount close to the expected Brillouin frequency shift, and launched into the opposite end of the sensing fibre to the other un-shifted beam. A strong Brillouin interaction results in a localized region of the fibre corresponding to zero delay between the counterpropagating beams of light. The peak Brillouin frequency corresponding to this localised region is obtained by scanning the frequency of the frequency shifter. By delaying the phase of one beam relative to the other, the position of the localised Brillouin interaction region can be swept along the sensing fibre. This is normally achieved by incorporating a delay fibre outside the sensing fibre, which essentially translates any change in the sweeping frequency of the laser into the phase change necessary to move the interrogation point along the sensing fibre. For the BOCDA based measurements, the spatial resolution $(\Delta \mathrm{z})$ and measurement range corresponding to the interval between correlation peaks $(\Delta \mathrm{d})$ are given by the following equations ${ }^{[2]}$ :

$\Delta \mathrm{z}=\frac{\Delta \mathrm{d} \cdot \Delta \mathrm{v}_{\mathrm{B}}}{\pi \cdot \Delta \mathrm{f}} \quad ; \quad \Delta \mathrm{d}=\frac{\mathrm{V}_{\mathrm{g}}}{2 \mathrm{f}_{\mathrm{m}}}$ 
where $\mathrm{f}_{\mathrm{m}}$ is the modulation frequency, $\Delta \mathrm{f}$ is the laser frequency excursion achieved when the laser wavelength is swept at frequency $f_{m}, \Delta v_{B}$ is the linewidth of the Brillouin spectrum and $v_{g}$ is the velocity of light in the fibre.

Previously, we reported the use of spontaneous Raman scattering to provide this temperature compensation, investigating separately heated and strained regions of fibre, but the spatial resolution was limited to $24 \mathrm{cms}$ [5] even with an averaging time of 40 minutes, due to the weak nature of Raman anti Stokes scattering. In order to more fully exploit the high spatial resolution capability of BOCDA, we investigated the benefit and robustness of combining the Brillouin anti-Stokes measurement, which is a stronger signal compared to Raman anti Stokes, with the Brillouin frequency based BOCDA technique. Using BOCDA and B-OTDR to discriminate temperature and strain requires the values of Brillouin frequency change obtained from BOCDA and intensity change from BOTDR for temperature and stain. This is expressed in the form of matrix, which on solving allows for the temperature compensated strain to be ascertained.

$\left[\begin{array}{l}\Delta v \\ \Delta \mathrm{I}_{\mathrm{B}_{A S}}\end{array}\right]=\left[\begin{array}{ll}\mathrm{C}_{\mathrm{BV}}^{\varepsilon} & \mathrm{C}_{\mathrm{BV}}^{\mathrm{T}} \\ \mathrm{C}_{\mathrm{BI}}^{\varepsilon} & \mathrm{C}_{\mathrm{BI}}^{\mathrm{T}}\end{array}\right]\left[\begin{array}{c}\Delta \varepsilon \\ \Delta \mathrm{T}\end{array}\right] \Rightarrow \quad \Delta \varepsilon=\frac{\mathrm{C}_{\mathrm{BI}}^{\mathrm{T}} \cdot \Delta v_{\mathrm{B}}-\mathrm{C}_{\mathrm{BV}}^{\mathrm{T}} \cdot \Delta \mathrm{I}_{\mathrm{B}_{A s}}}{\mathrm{C}_{\mathrm{BV}}^{\varepsilon} \mathrm{C}_{\mathrm{BI}}^{\mathrm{T}}-\mathrm{C}_{\mathrm{BI}}^{\varepsilon} \mathrm{C}_{\mathrm{Bv}}^{\mathrm{T}}}$

This equation relies on the assumption that the coefficients describing the change in frequency or power with strain or temperature are constant as the other parameter is varied. The validity of this assumption is the subject of a separate investigation to be reported. The temperature compensated strain resolution can be estimated from equation 2 using previously determined experimental values of Brillouin coefficients ${ }^{[1,6]}$.

$|\delta(\Delta \varepsilon)|=\frac{\left|\mathrm{C}_{\mathrm{BI}}^{\mathrm{T}}\right|\left|\delta \Delta v_{\mathrm{B}}\right|+\left|\mathrm{C}_{\mathrm{Bv}}^{\mathrm{T}} \| \delta \Delta \mathrm{I}_{\mathrm{B}_{A S S}}\right|}{\left|\mathrm{C}_{\mathrm{Bv}}^{\varepsilon} \mathrm{C}_{\mathrm{BI}}^{\mathrm{T}}-\mathrm{C}_{\mathrm{BI}}^{\varepsilon} \mathrm{C}_{\mathrm{Bv}}^{\mathrm{T}}\right|}$

$\delta \Delta \mathrm{Y}_{\mathrm{B}}$ and $\delta \Delta \mathrm{I}_{\mathrm{B}_{\triangle \mathrm{S}}}$ are the RMS errors on the Brillouin frequency and anti-Stokes intensity measurements respectively.

\section{EXPERIMENT}

The experimental set up, with detail of the sensing fiber layout is shown in fig. 1.

(a)

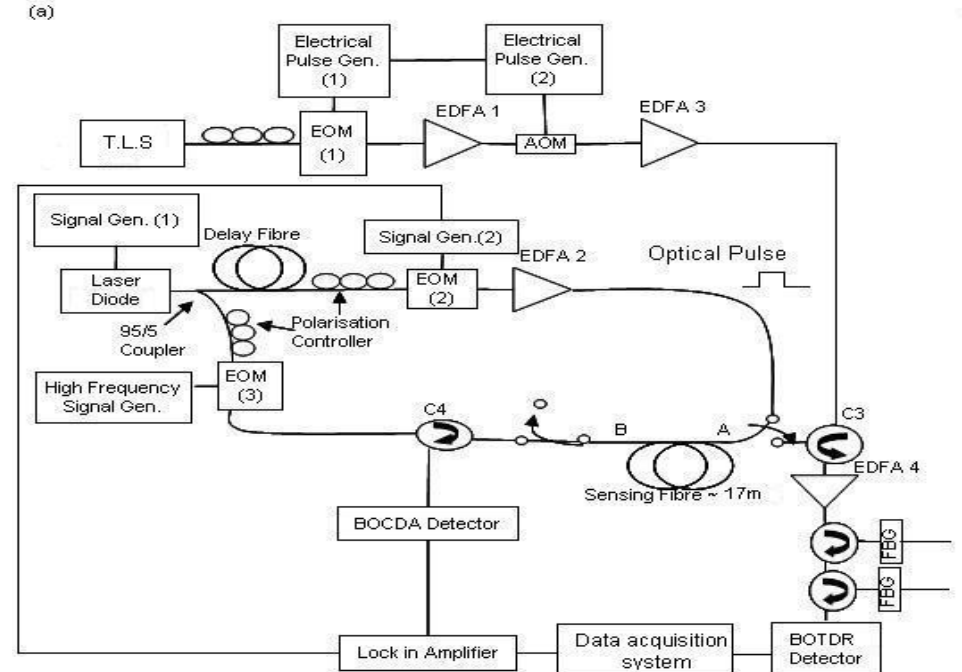

(b)

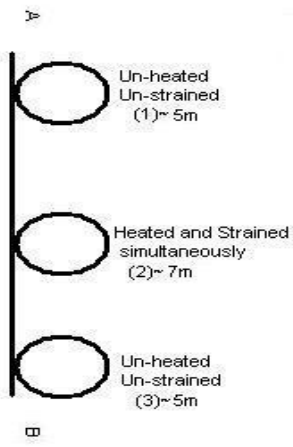

m

Figure 1. (a) The experimental configuration for a temperature compensated distributed strain sensor system; (b) Layout of the $17 \mathrm{~m}$ sensing fibre.

The sensing fiber comprised $17 \mathrm{~m}$ of standard single mode fiber with an effective area $~ 80 \mu \mathrm{m}^{2}$, loss $\sim 0.20 \mathrm{~dB} / \mathrm{km}$ and dispersion $\sim 17 \mathrm{ps} / \mathrm{km} \mathrm{nm}$ at $1550 \mathrm{~nm}$. The injection current of the laser diode (linewidth $\sim 5 \mathrm{MHz}, 1550 \mathrm{~nm}$ ) was modulated to achieve a frequency excursion $\Delta \mathrm{f}$ of $8.8 \mathrm{GHz}$ with sinusoidal sweep rates $\mathrm{f}_{\mathrm{m}}$ of $2.14504 \mathrm{MHz}-2.1466 \mathrm{MHz}$ (frequency step $0.03 \mathrm{kHz}$ ) in order to enable a single correlation peak to be scanned over the fibers from section 1, 2 and 3 respectively. The output from a tuneable laser source (T.L.S) linewidth $\sim 100 \mathrm{KHz}, 1534 \mathrm{~nm}$, was fed through a cascaded arrangement of electro-optic modulator (EOM), erbium doped fiber amplifier (EDFA) and an acousto-optic modulator (AOM), resulting in $0.3-0.5 \mathrm{~ns}, 5 \mathrm{~W}$ peak power pulses, which were subsequently launched into the $131 \mathrm{~m}$ sensing fiber. The spontaneous Brillouin anti-Stokes back-scattered signal was 
amplified using a $30 \mathrm{~dB}$ gain optical amplifier and filtered using two narrow $(\sim 3.5 \mathrm{GHz})$ fibre Bragg gratings.

The amplified signal was detected using a $200 \mathrm{MHz}$ APD.

\section{RESULTS AND DISCUSSION}

Figure 2, shows a summary of the BOCDA results. Figure 2(a), shows the two Brillouin gain spectra (BGS) corresponding to three different sections of the sensing fiber. The black trace shows the BGS corresponding to section 1 and 3, which remained un-heated and un-strained, while the red trace corresponds to section 2 which was simultaneously subjected to a temperature of $70^{\circ} \mathrm{C}$ and a nominal strain of $1285 \mu \varepsilon$. Fig. 2 (b) shows the change in Brillouin frequency shift of $101 \mathrm{MHz}$ emerging as the correlation peak is swept from section 1 to section 2.

(a)

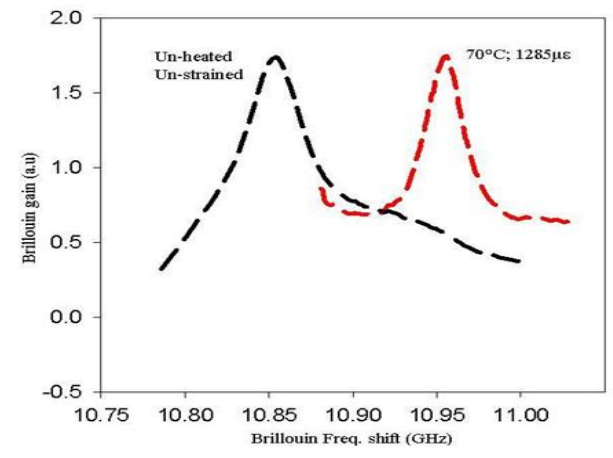

(b)

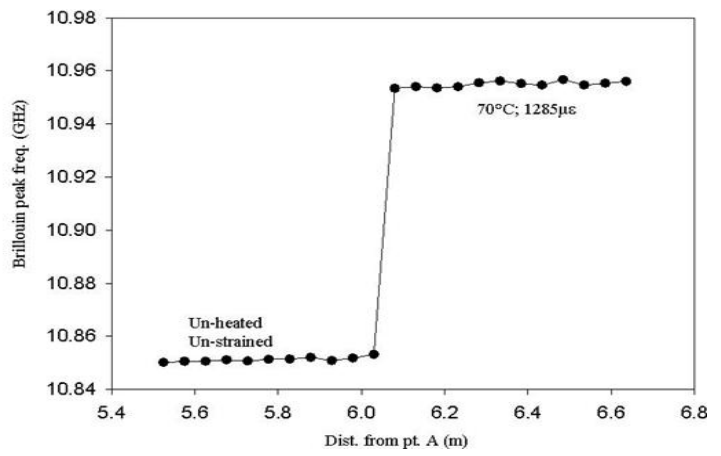

Figure 2. (a) The Brillouin peak shown in black trace corresponds to the un-heated, un-strained sections of $5 \mathrm{~m}$ each, while the Brillouin peak shown in red represents the $7 \mathrm{~m}$ section of fiber simultaneously subjected to a temperature of $70^{\circ} \mathrm{C}$ and a nominal strain of $1285 \mu \varepsilon$; (b) The step change in Brillouin peak frequency when scanning the correlation peak from the un-heated, un-strained section to the section of fiber simultaneously subjected to a temperature of $70^{\circ} \mathrm{C}$ and a nominal strain of $1285 \mu \varepsilon$.

The measured spatial resolution, figure 2(b), equalled the expected theoretical value of $5 \mathrm{~cm}$ with a frequency resolution of $1 \mathrm{MHz}$.

Figure 3 depicts a normalized B-OTDR plot with the insert showing a spatial resolution of $5 \mathrm{cms}$.

The spatial resolution was evaluated using the 90/10 response of a step change in temperature, between the un-heated $\left(23.6^{\circ} \mathrm{C}\right)$, un-strained section 1 and the simultaneously heated $\left(70^{\circ} \mathrm{C}\right)$ and strained $(1285 \mu \varepsilon)$ section 2 . The r.m.s intensity noise $\left(\delta \Delta \mathrm{I}_{\mathrm{B}_{A S}}\right)$ of the heated section, of Fig. 3 was measured to be $0.71 \%$, which translates into a temperature resolution of $1.9^{\circ} \mathrm{C}$, in the absence of strain uncertainty. The frequency error $\left(\delta \Delta v_{B}\right)$ of $1 \mathrm{MHz}$ on the Brillouin frequency based strain measurements corresponds to a strain resolution of $21 \mu \varepsilon$ in the absence of temperature uncertainty. To ascertain the strain resolution under conditions where the temperature effects are also present, equation 3 is used to compute the strain resolution. The combined errors of the Brillouin frequency, $\delta \Delta v_{\mathrm{B}}=1 \mathrm{MHz}$ and Brillouin intensity, $\delta \Delta \mathrm{I}_{\mathrm{B}_{\mathrm{AS}}}=0.71 \%$ with coefficients $\mathrm{C}_{\mathrm{Bv}}^{\varepsilon}=4.5 \mathrm{MHz} / 100 \mu \varepsilon ; \quad \mathrm{C}_{\mathrm{Bv}}^{\mathrm{T}}=1 \mathrm{MHz} /{ }^{\circ} \mathrm{C} ; \mathrm{C}_{\mathrm{BI}}^{\mathrm{T}}=0.33 \% /{ }^{\circ} \mathrm{C} ; \quad \mathrm{C}_{\mathrm{BI}}^{\varepsilon}=-8.4 \times 10^{-4} \% / \mu \varepsilon$, yield a temperature compensated strain resolution of $66 \mu \varepsilon$ for a strain sensor of $17 \mathrm{~m}$ long with a sub metre spatial resolution of $5 \mathrm{~cm}$. 


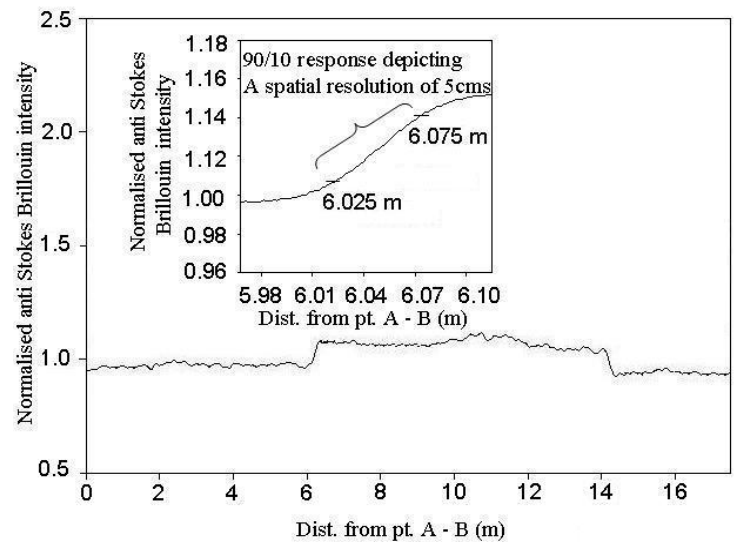

Fig. 3 The normalised B-OTDR plot of the $17 \mathrm{~m}$ sensing fiber, with the insert showing a 90/10 step response for the transition from unheated, unstrained section (1) to the simultaneously heated $\left(70^{\circ} \mathrm{C}\right)$ and nominally strained $(1285 \mu \varepsilon)$ section $(2)$ of the sensing fibre, indicating a spatial resolution of $5 \mathrm{cms}$.

\section{CONCLUSION}

A high spatial resolution temperature compensated strain sensor has been demonstrated using BOCDA combined with BOTDR. With the use of the stronger Brillouin anti-Stokes signal as compared to the previously used Raman anti-Stokes, the signal to noise for intensity based temperature measurements was improved nearly 2.3 times i.e., from $1.6 \%{ }^{[4]}$ to $0.71 \%$. This permitted both the strain and spatial resolutions of the temperature compensated strain to be reduced from $97 \mu \varepsilon$ and $24 \mathrm{cms}{ }^{[9]}$ to $66 \mu \varepsilon$ and $5 \mathrm{cms}$. The time domain trace was averaged $2^{16}$ times and collected using an improved data acquisition system which reduced the data collection time from 40 minutes to a little under 1 minute. This potentially provides new opportunities for monitoring cyclic loading of smart composite structures which often undergo considerable heating during the strain cycles.

\section{References}

[1] Maughan S.M., Kee H.H. and Newson T.P., "Simultaneous distributed fibre temperature and strain sensor using microwave coherent detection of spontaneous Brillouin backscatter', Meas. Sci. Technol. 12 834-842 (2001)

[2] Song K.Y., He Z. and Hotate K., "Distributed strain measurement with millimeter-order spatial resolution based on Brillouin optical correlation domain analysis", Opt. Lett. 31, 2526-2528 (2006)

[3] Alahbabi M.N., Cho Y.T. and Newson T.P., "Long-range distributed temperature and strain optical fibre sensor based on the coherent detection of spontaneous Brillouin scattering with in-line Raman amplification", Meas. Sci. Technol. 17, 1082-1090 (2006)

[4] Alahbabi M.N., Lawrence N.P., Cho Y.T. and Newson T.P., "High spatial resolution microwave detection system for Brillouin-based distributed temperature and strain sensors", Meas. Sci. Technol. 15, 1539-1543 (2004)

[5] Belal M., Cho Y.T., Ibsen M. and Newson T.P., " A temperature compensated high spatial resolution distributed strain sensor', Meas. Sci. Technol. 21 (2010)

[6] De Souza K., Wait P.C. and Newson T.P., "Characterisation of the strain dependence of the Landau Placzek ratio for distributed sensing." Elec. lett. 33 (7) 615 - 616 (1997) 